\title{
Accessing linearly polarized gluon distribution in $J / \psi$ production at the electron-ion collider
}

\author{
Raj Kishore and Asmita Mukherjee \\ Department of Physics, Indian Institute of Technology Bombay, Mumbai-400076, India
}

(Received 27 November 2018; published 18 March 2019)

\begin{abstract}
We calculate the $\cos 2 \phi_{h}$ asymmetry in $J / \psi$ production in electron-proton collision for the kinematics of the planned electron-ion collider (EIC). This directly probes the Weiszäcker-Williams type linearly polarized gluon distribution. Assuming generalized factorization, we calculate the asymmetry at next-toleading-order when the energy fraction of the $J / \psi$ satisfies $z<1$ and the dominating subprocess is $\gamma^{*}+g \rightarrow c+\bar{c}+g$. We use the nonrelativistic QCD based color singlet model for $J / \psi$ production. We investigate the small $x$ region which will be accessible at the EIC. We present the upper bound of the asymmetry, as well as estimate it using a (i) Gaussian-type parametrization for the transverse momentum dependent parton distributions and (ii) McLerran-Venugopalan model at small $x$. We find small but sizable asymmetry in all three cases.
\end{abstract}

DOI: 10.1103/PhysRevD.99.054012

\section{INTRODUCTION}

$J / \psi$ electroproduction is a direct probe of gluon transverse momentum dependent parton distributions (TMDs), as the leading process is the virtual photon-gluon fusion. Very little is known so far about the gluon TMDs [1], apart from a positivity bound. Recently, unpolarized TMD gluon parton distributions (PDFs) have been extracted from LHCb data [2]. TMD pdfs are process dependent due to the initial and/or final state interactions, in other words, due to the presence of gauge links in their operator definitions. Each gluon TMD contains two gauge links in contrast to the quark TMDs that contain one. Because of this, the process dependence of gluon TMDs is more involved than quark TMDs [3]. The simplest possible configurations are both future pointing $[++]$ or one future and one past pointing $[+-]$ gauge links. In the literature related to small$x$ physics, the former is called Weizsäcker-Williams (WW) gluon distribution [4,5]. For unpolarized gluons, WW gluon distribution can be interpreted as the number density of gluons inside hadrons in light-cone gauge. The other distribution, $[+-]$, is called dipole distribution [6]. This appears in many physical processes and is the Fourier transform of the color dipole amplitude $[7,8]$. In small $x$ physics, these two types of unintegrated gluon distributions have been discussed in the literature quite extensively [917]. Apart from the unpolarized gluon TMD, the linearly

Published by the American Physical Society under the terms of the Creative Commons Attribution 4.0 International license. Further distribution of this work must maintain attribution to the author(s) and the published article's title, journal citation, and DOI. Funded by SCOAP ${ }^{3}$. polarized gluon TMD recently has attracted quite a lot of interest. This basically measures an interference between an amplitude when the active gluon is polarized along the $x$ (or $y$ ) direction and a complex conjugate amplitude with the gluon polarized in the $y$ (or $x$ ) direction in an unpolarized hadron [6]. This was introduced for the first time in [1] and calculated in a model in [18]. It has been shown that the linearly polarized gluon distribution affects the unpolarized cross section of scattering processes, as well as an azimuthal asymmetry of the type $\cos 2 \phi_{h}$ [19]. The linearly polarized gluon distribution is a time reversal even (T even) object, and can be WW type or dipole type, depending on the gauge links. $J / \psi$ production in $e p$ collision probes the WW-type linearly polarized gluon TMD through a virtual photon-gluon fusion process. The leading order (LO) process $\gamma^{*}+g \rightarrow c \bar{c} \rightarrow J / \psi$ contributes to the asymmetry at $z=1$ [20]. The linearly polarized gluon distribution has not been extracted from data yet. However, there are quite a large amount of theoretical studies about how to probe it in different experiments. In [21] the authors have proposed to probe it in dijet imbalance in the unpolarized hadronic collision and also in heavy quark pair production in $e p$ collision and in $p p$ collision $[15,19,22,23]$. It can also be probed in quarkonium pair production in $p p$ collision [2], and in associated production of a dilepton and $J / \psi$ [24]. Very recently, in [25] the authors have investigated the possibility to probe it in dijet imbalance in $e A$ collision. $h_{1}^{\perp g}$ affects the transverse momentum distribution of the final state hadron like Higgs boson [26-29] and heavy quarkonium [30-32] in unpolarized $p p$ collision. Although $h_{1}^{\perp g}$ can be probed in $p p$ and $p A$ collision, initial and final state interactions may affect 
the factorization in such processes. Such complications are less in $e p$ collision processes for example at the electronion collider (EIC). In a previous work [20] we have investigated the possibility of probing $h_{1}^{\perp g}$ in $\cos 2 \phi$ asymmetry in $J / \psi$ production through the leading order (LO) process $\gamma^{*}+g \rightarrow J / \psi$ at the future EIC. This $2 \rightarrow 1$ process contributes at $z=1$, where $z$ is the energy fraction of the photon carried by the $J / \psi$ in the proton rest frame. Here, we extend our analysis to the kinematical region $z<1$. We consider the unpolarized $e P$ collision. The production mechanism of $J / \psi$ is not yet well understood theoretically. The most widely used approach is based on nonrelativistic QCD (NRQCD) [33]. Here one assumes a factorization of the amplitude into a hard part where the $c \bar{c}$ pair is produced perturbatively in the process $\gamma^{*}+g \rightarrow c+\bar{c}+g$. The heavy quark pair then hadronizes to form the $J / \psi$ bound state. The hadronization is described in terms of the long distance matrix elements (LDMEs) which are obtained by fitting the data. For some LDMEs lattice calculations are available. They have definite scaling properties with respect to the velocity parameter $v$, which is assumed to be small. The cross section for the production of $J / \psi$ is expressed as a double expansion in terms of the strong coupling constant $\alpha_{s}$ as well as $v$ [34]. For $J / \psi, v \approx 0.3$. In NRQCD, the heavy quark pair can be produced both in the color singlet (CS) state [35-38] or in the color octet (CO) state [39-41]. The former is called the CS model and the latter is the CO model. In the CS model, the heavy quark pair is produced in the hard process as a color singlet with the same quantum number as $J / \psi$. In [42] the $J / \psi$ production rate for unpolarized $p p$ collision at RHIC assuming a generalized TMD factorization was calculated in the CS model, and it was found that the theoretical estimate reasonably explains the data for low values of $p_{T}$, where $p_{T}$ is the transverse momentum of $J / \psi$. However, high $p_{T}$ spectra for $J / \psi$ production needs the inclusion of $\mathrm{CO}$ states. As we showed in [43] both $\mathrm{CS}$ and $\mathrm{CO}$ contributions are needed to match the HERA data. However, in this work, as a first study, we calculate the $\cos 2 \phi$ asymmetry in $J / \psi$ production in $e p$ collision in the CS model. All previous studies of this asymmetry in $e P$ collision have considered the LO process. In this work, for the first time, we investigate the asymmetry in the kinematical region $z<1$. As we are interested in the small $x$ region, we consider the process $\gamma^{*}+g \rightarrow J / \psi+g$, as gluon distributions are dominant at small $x$. This process probes the WW-type gluon TMDs.

In order to estimate the $\cos 2 \phi$ asymmetry, we use three different models for the TMDs. First, we use a Gaussian parametrization [30-32] for both the linearly polarized gluon distribution and the unpolarized TMD. The linearly polarized gluons satisfy an upper bound and the asymmetry reaches its maximum value when this upper bound is saturated. We also calculate the upper bound of the asymmetry. Finally, in the small $x$ region, the WW-type gluon distributions are calculated using a saturation model [44-46]. TMDs in the McLerran-Venugopalan (MV) model, although expected to work better for a large nucleus, have been found to be phenomenologically successful for the nucleon [47]. We have used a regulated MV model in the small $x$ region for the WW-type gluon TMDs. We have compared the asymmetry in all three cases in the kinematics of the planned electron-ion collider (EIC).

The paper is organized into four sections starting with the Introduction in Sec. I. In Sec. II, we provide the analytic framework, kinematics of the process and the calculations of asymmetry in different models. We provide the numerical estimations in Sec. III and conclude the results in Sec. IV. Some detailed analytic results are given in the Appendix.

\section{FRAMEWORK FOR CALCULATION}

The process we have considered here is

$$
e(l)+p(P) \rightarrow e\left(l^{\prime}\right)+J / \psi\left(P_{h}\right)+X .
$$

Both the scattering electron and target proton are unpolarized. Four momentum of particles is represented within the round brackets. The dominating subprocess for small $x$ for quarkonium production in $e p$ collision is the photon-gluon fusion process, at leading order this process contributes at $z=1$ [20]. In this work, we consider the next-to-leading-order (NLO) process $\gamma^{*}(q)+g(k) \rightarrow$ $J / \psi\left(P_{h}\right)+g\left(p_{g}\right)$ and the kinematical region $z<1$, which will be accessible at EIC. The final state gluon is not detected. Here the variable $z$ is defined as $z=P \cdot P_{h} / P \cdot q$ which is the energy fraction of $J / \psi$ in the proton rest frame. We use a generalized factorization scheme taking into account the partonic transverse momenta. We consider the frame in which the virtual photon and proton are moving in the $+z$ and the $-z$ direction respectively. The incoming and outgoing electrons form a lepton plane, which provides a reference for measuring azimuthal angles of other particles. The four momenta of proton and virtual photon $q=l-l^{\prime}$ are given by [20]

$$
\begin{gathered}
P=n_{-}+\frac{M_{p}^{2}}{2} n_{+} \approx n_{-} \\
q=-x_{B} n_{-}+\frac{Q^{2}}{2 x_{B}} n_{+} \approx-x_{B} P+(P \cdot q) n_{+},
\end{gathered}
$$

where $Q^{2}=-q^{2}$ and Bjorken variable, $x_{B}=\frac{Q^{2}}{2 P \cdot q} . M_{p}$ is the mass of proton. All four momenta are written in terms of lightlike vectors $n_{-}=P$ and $n_{+}=n=\left(q+x_{B} P\right) / P \cdot q$, such that $n_{+} \cdot n_{-}=1$ and $n_{-}^{2}=n_{+}^{2}=0$. The leptonic momenta can be written as 


$$
\begin{aligned}
l & =\frac{1-y}{y} x_{B} P+\frac{1}{y} \frac{Q^{2}}{2 x_{B}} n+\frac{\sqrt{(1-y)}}{y} Q \hat{l}_{\perp} \\
& =\frac{1-y}{y} x_{B} P+\frac{s}{2} n+\frac{\sqrt{(1-y)}}{y} Q \hat{l}_{\perp} \\
l^{\prime} & =\frac{1}{y} x_{B} P+\frac{1-y}{y} \frac{Q^{2}}{2 x_{B}} n+\frac{\sqrt{(1-y)}}{y} Q \hat{l}_{\perp} \\
& =\frac{1}{y} x_{B} P+(1-y) \frac{s}{2} n+\frac{\sqrt{(1-y)}}{y} Q \hat{l}_{\perp} .
\end{aligned}
$$

Here, $s=(l+P)^{2}=2 P \cdot l$ is the center of mass energy of electron-proton scattering. $y=P \cdot q / P \cdot l$, such that the relation $Q^{2}=s x_{B} y$ holds. The virtual photon and target proton system invariant mass squared is defined as $W^{2}=(P+q)^{2}$. In terms of the lightlike vectors defined above, the four momenta of initial state gluon is given as

$$
k=x P+k_{\perp}+\left(p \cdot P-x M_{p}^{2}\right) n \approx x P+k_{\perp},
$$

where $x=k \cdot n$ is the light-cone momentum fraction. The four momentum of the final state $J / \psi$ and the final state gluon are given by

$$
\begin{gathered}
P_{h}=z(P \cdot q) n+\frac{M^{2}+\mathbf{P}_{h \perp}^{2}}{2 z P \cdot q} P+P_{h \perp} \\
p_{g}=(1-z)(P \cdot q) n+\frac{\mathbf{p}_{g \perp}^{2}}{2(1-z) P \cdot q} P+p_{g \perp},
\end{gathered}
$$

$P_{h}^{2}=-\mathbf{P}_{h \perp}^{2} . M$ is the mass of $J / \psi$.

For the partonic level process, $\gamma^{*}(q)+g(k) \rightarrow J / \psi\left(P_{h}\right)+$ $g\left(p_{g}\right)$, we can define the Mandelstam variables as follows:

$$
\begin{aligned}
\hat{s} & =(k+q)^{2}=q^{2}+2 k \cdot q=\frac{x Q^{2}}{x_{B}}-Q^{2}, \\
\hat{t} & =\left(k-P_{h}\right)^{2}=M^{2}-2 k \cdot P_{h} \\
& =M^{2}-\frac{x z Q^{2}}{x_{B}}+2 k_{\perp} P_{h \perp} \cos \left(\phi-\phi_{h}\right), \\
\hat{u} & =\left(q-P_{h}\right)^{2}=M^{2}+q^{2}-2 q \cdot P_{h} \\
& =M^{2}-(1-z) Q^{2}-\frac{M^{2}+P_{h \perp}^{2}}{z} .
\end{aligned}
$$

The $\phi$ and $\phi_{h}$ are the azimuthal angles of the initial gluon and $J / \psi$ transverse momentum vector respectively.

We use a framework based on the generalized parton model approach with the inclusion of intrinsic transverse momentum effects, and assume TMD factorization. The differential cross section for the unpolarized process is given by [20]

$$
\begin{aligned}
d \sigma= & \frac{1}{2 s} \frac{d^{3} l^{\prime}}{(2 \pi)^{3} 2 E_{l^{\prime}}} \frac{d^{3} P_{h}}{(2 \pi)^{3} 2 E_{P_{h}}} \int \frac{d^{3} p_{g}}{(2 \pi)^{3} 2 E_{g}} \\
& \times \int d x d^{2} \boldsymbol{k}_{\perp}(2 \pi)^{4} \delta\left(q+k-P_{h}-p_{g}\right) \\
& \times \frac{1}{Q^{4}} L^{\mu \mu^{\prime}}(l, q) \Phi^{\nu \nu^{\prime}}\left(x, \boldsymbol{k}_{\perp}\right) \mathcal{M}_{\mu \nu}^{\gamma^{*}+g \rightarrow J / \psi+g} \mathcal{M}_{\mu^{\prime} \nu^{\prime}}^{* \gamma^{*}+g \rightarrow J / \psi+g},
\end{aligned}
$$

where $L^{\mu \mu^{\prime}}$ is the leptonic tensor which is given by

$$
L^{\mu \mu^{\prime}}(l, q)=e^{2}\left(-g^{\mu \mu^{\prime}} Q^{2}+2\left(l^{\mu} l^{\mu^{\prime}}+l^{\mu^{\prime}} l^{\prime \mu}\right)\right)
$$

with $e$ the electric charge of electron. $\Phi^{\nu \nu^{\prime}}$ is the gluon correlator which can be parametrized in terms of gluon TMDs. For an unpolarized proton, at leading twist, the gluon correlator can be given as [1]

$$
\begin{aligned}
\phi_{g}^{\nu \nu^{\prime}}\left(x, \mathbf{k}_{\perp}\right)= & \frac{1}{2 x}\left[-g_{\perp}^{\nu \nu^{\prime}} f_{1}^{g}\left(x, \mathbf{k}_{\perp}^{2}\right)\right. \\
& \left.+\left(\frac{k_{\perp}^{\nu} k_{\perp}^{\nu^{\prime}}}{M_{p}^{2}}+g_{\perp}^{\nu \nu^{\prime}} \frac{\mathbf{k}_{\perp}^{2}}{2 M_{p}^{2}}\right) h_{1}^{\perp g}\left(x, \mathbf{k}_{\perp}^{2}\right)\right],
\end{aligned}
$$

where $f_{1}^{g}\left(x, \boldsymbol{k}_{\perp}^{2}\right)$ is the unpolarized gluon distribution and $h_{1}^{\perp g}\left(x, \boldsymbol{k}_{\perp}^{2}\right)$ is the linearly polarized gluon distribution. $g_{\perp}^{\nu \nu^{\prime}}=g^{\nu \nu^{\prime}}-P^{\nu} n^{\nu^{\prime}} / P \cdot n-P^{\nu^{\prime}} n^{\nu} / P \cdot n$.

\section{A. $J / \psi$ production in NRQCD based color singlet (CS) framework}

The dominating subprocess at is $\gamma^{*}+g \rightarrow J / \psi+g$. All the tree level Feynman diagrams corresponding to this process are given in Fig. 1.

The general expression of the amplitude for the bound state production of $J / \psi$ in NRQCD framework can be written as [20,30]

$$
\begin{aligned}
\mathcal{M} & \left(\gamma^{*} g \rightarrow Q \bar{Q}\left[2 S+1 L_{J}^{(1)}\right]\left(P_{h}\right)+g\right) \\
= & \sum_{L_{z} S_{z}} \int \frac{d^{3} \boldsymbol{k}^{\prime}}{(2 \pi)^{3}} \Psi_{L L_{z}}\left(\boldsymbol{k}^{\prime}\right)\left\langle L L_{z} ; S S_{z} \mid J J_{z}\right\rangle \\
& \times \operatorname{Tr}\left[O\left(q, k, P_{h}, k^{\prime}\right) \mathcal{P}_{S S_{z}}\left(P_{h}, k^{\prime}\right)\right] .
\end{aligned}
$$

As we have imposed a cutoff on $z, z<0.9$, we do not need to consider the virtual diagrams as they contribute at $z=1$. In the above equation, $2 k^{\prime}$ is the relative momentum of heavy quarks and $O\left(q, k, P_{h}, k^{\prime}\right)$ is calculated from the Feynman diagrams. The spinors of heavy quark, antiquark legs are absorbed into the bound state wave function. By considering the contribution from all the Feynman diagrams, $O\left(q, k, P_{h}, k^{\prime}\right)$ is given by 

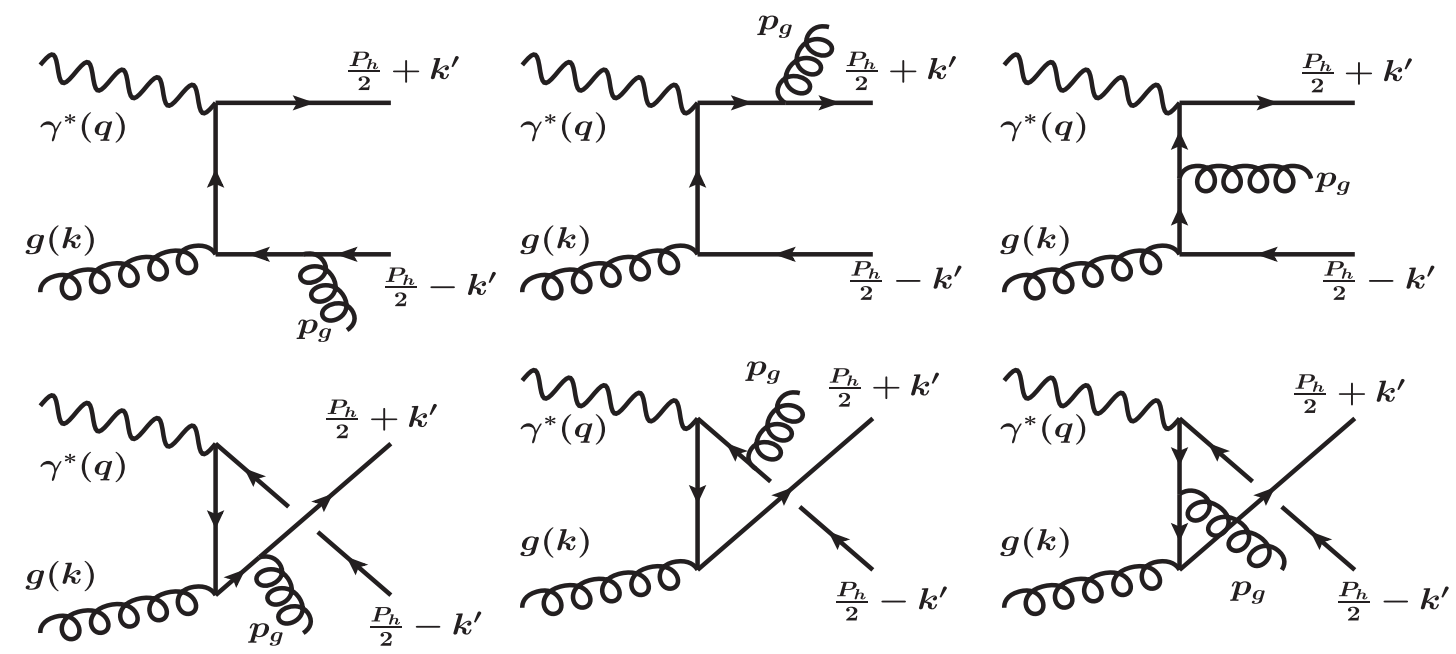

FIG. 1. Feynman diagrams for the $\gamma^{*}+g \rightarrow J / \psi+g$ process.

$$
O\left(q, k, P_{h}, k^{\prime}\right)=\sum_{m=1}^{6} \mathcal{C}_{m} O_{m}\left(q, k, P_{h}, k^{\prime}\right),
$$

where, $O_{m},(m=1,2, \ldots, 6)$ are corresponding to each Feynman diagram and $\mathcal{C}_{m}$ represents the color factor of corresponding diagram. The expressions for $O_{m}$ are given below:

$$
\begin{aligned}
O_{1}= & 4 g_{s}^{2}\left(e e_{c}\right) \varepsilon_{\lambda_{g}}^{\rho *}\left(p_{g}\right) \gamma_{\nu} \frac{\not P_{h}+2 \not \not^{\prime}-2 \not 1+M}{\left(P_{h}+2 k^{\prime}-2 q\right)^{2}-M^{2}} \\
& \times \gamma_{\mu} \frac{-\not p_{h}+2 \not k^{\prime}-2 \not \not_{g}+M}{\left(P_{h}-2 k^{\prime}+2 p_{g}\right)^{2}-M^{2}} \gamma_{\rho},
\end{aligned}
$$

$$
\begin{aligned}
O_{2}= & 4 g_{s}^{2}\left(e e_{c}\right) \varepsilon_{\lambda_{g}}^{\rho^{*}}\left(p_{g}\right) \gamma_{\rho} \frac{\not p_{h}+2 \not k^{\prime}+2 \not \not g_{g}+M}{\left(P_{h}+2 k^{\prime}+2 p_{g}\right)^{2}-M^{2}} \\
& \times \gamma_{\nu} \frac{-\not p_{h}+2 \not k^{\prime}+2 \not k+M}{\left(P_{h}-2 k^{\prime}-2 k\right)^{2}-M^{2}} \gamma_{\mu},
\end{aligned}
$$

$$
\begin{aligned}
O_{3}= & 4 g_{s}^{2}\left(e e_{c}\right) \varepsilon_{\lambda_{g}}^{\rho *}\left(p_{g}\right) \gamma_{\nu} \frac{\not P_{h}+2 \not k^{\prime}-2 \not 1+M}{\left(P_{h}+2 k^{\prime}-2 q\right)^{2}-M^{2}} \\
& \times \gamma_{\rho} \frac{-\not P_{h}+2 \not \ell^{\prime}+2 \not k+M}{\left(P_{h}-2 k^{\prime}-2 k\right)^{2}-M^{2}} \gamma_{\mu} .
\end{aligned}
$$

Here, the mass of bound state $M$ is assumed to be twice the mass of charm quark $\left(m_{c}\right)$, i.e. $M=2 m_{c}$. The charge conjugation invariance allows us to write the expressions for $\left(O_{4}, O_{5}\right.$ and $\left.O_{6}\right)$, from the other Feynman diagrams, by reversing the fermion line and replacing $k^{\prime}$ by $-k^{\prime}$. Assuming the $Q \bar{Q}$ is formed in the color singlet state, the color factor of each diagram is given by

$$
\begin{aligned}
& \mathcal{C}_{1}=\mathcal{C}_{5}=\mathcal{C}_{6}=\sum_{i j}\langle 3 i ; \overline{3} j \mid 1\rangle\left(t_{a} t_{b}\right)_{i j}, \\
& \mathcal{C}_{2}=\mathcal{C}_{3}=\mathcal{C}_{4}=\sum_{i j}\langle 3 i ; \overline{3} j \mid 1\rangle\left(t_{b} t_{a}\right)_{i j} .
\end{aligned}
$$

The SU(3) Clebsch-Gordan coefficients for CS are given by

$$
\langle 3 i ; \overline{3} j \mid 1\rangle=\frac{\delta^{i j}}{\sqrt{N_{c}}},
$$

where $N_{c}$ is the number of colors. The generators of the $\mathrm{SU}(3)$ group satisfy the relations $\operatorname{Tr}\left(t_{a}\right)=0, \operatorname{Tr}\left(t_{a} t_{b}\right)=$ $\delta_{a b} / 2$ and $\operatorname{Tr}\left(t_{a} t_{b} t_{c}\right)=\frac{1}{4}\left(d_{a b c}+i f_{a b c}\right)$.

From these relations, we get the color factor for the production of the $Q \bar{Q}$ pair in the CS state as follows:

$$
\mathcal{C}_{1}=\mathcal{C}_{2}=\mathcal{C}_{3}=\mathcal{C}_{4}=\mathcal{C}_{5}=\mathcal{C}_{6}=\frac{\delta_{a b}}{2 \sqrt{N_{c}}}
$$

The spin projection operator, given in the equation of amplitude of the bound state, includes the spinors of heavy quark and antiquark and is given by [30]

$$
\begin{aligned}
& \mathcal{P}_{S S_{z}}\left(P_{h}, k^{\prime}\right) \\
& \quad=\sum_{s_{1} S_{2}}\left\langle\frac{1}{2} s_{1} ; \frac{1}{2} s_{2} \mid S S_{z}\right\rangle v\left(\frac{P_{h}}{2}-k^{\prime}, s_{1}\right) \bar{u}\left(\frac{P_{h}}{2}+k^{\prime}, s_{2}\right) \\
& \quad=\frac{1}{4 M^{3 / 2}}\left(-\not P_{h}+2 \not k^{\prime}+M\right) \Pi_{S S_{z}}\left(\not P_{h}+2 \not k^{\prime}+M\right)+\mathcal{O}\left(k^{\prime 2}\right),
\end{aligned}
$$

where $\Pi_{S S_{z}}=\gamma^{5}$ for the singlet $(S=0)$ state and $\Pi_{S S_{z}}=$ $\phi_{s_{z}}\left(P_{h}\right)$ for the triplet $(S=1)$ state. $\varepsilon_{S_{z}}\left(P_{h}\right)$ is the spin polarization vector of the $Q \bar{Q}$ pair. Since, $k^{\prime} \ll P_{h}$, one can perform Taylor expansion of the amplitude around $k^{\prime}=0$. In that expansion, the first term gives the $S$-waves 
$(\mathrm{L}=0, \mathrm{~J}=0,1)$. For the $P$-waves $(1=1, \mathrm{~J}=0,1,2)$, we need to consider the linear terms in $k^{\prime}$ in the expansion as the radial wave function $R_{1}(0)=0$ for $P$-wave. Since, $J / \psi$ is a ${ }^{3} S_{1}$ state, in the color singlet model we calculate the contribution of the CS state ${ }^{3} S_{1}$ :

$$
\begin{aligned}
\mathcal{M} & \left.{ }^{2 S+1} S_{J}^{(1)}\right]\left(P_{h}, k\right) \\
& =\left.\frac{1}{\sqrt{4 \pi}} R_{0}(0) \operatorname{Tr}\left[O\left(q, k, P_{h}, k^{\prime}\right) \mathcal{P}_{S S_{z}}\left(P_{h}, k^{\prime}\right)\right]\right|_{k^{\prime}=0} \\
& =\frac{1}{\sqrt{4 \pi}} R_{0}(0) \operatorname{Tr}\left[O(0) \mathcal{P}_{S S_{z}}(0)\right],
\end{aligned}
$$

where

$$
\begin{aligned}
O(0) & =\left.O\left(q, k, P_{h}, k^{\prime}\right)\right|_{k^{\prime}=0}, \\
\mathcal{P}_{S S_{z}}(0) & =\left.\mathcal{P}_{S S_{z}}\left(P_{h}, k^{\prime}\right)\right|_{k^{\prime}=0} .
\end{aligned}
$$

We have the following symmetry relations for the ${ }^{3} S_{1}$ state:

$$
\begin{aligned}
& \operatorname{Tr}\left[O_{1}(0)\left(-\not \not_{h}+M\right) \phi_{s_{z}}\right]=\operatorname{Tr}\left[O_{4}(0)\left(-\not \not_{h}+M\right) \phi_{s_{z}}\right] \\
& \operatorname{Tr}\left[O_{2}(0)\left(-\not \not_{h}+M\right) \phi_{s_{z}}\right]=\operatorname{Tr}\left[O_{5}(0)\left(-\not \not_{h}+M\right) \phi_{s_{z}}\right] \\
& \operatorname{Tr}\left[O_{3}(0)\left(-\not \not_{h}+M\right) \phi_{s_{z}}\right]=\operatorname{Tr}\left[O_{6}(0)\left(-\not \not_{h}+M\right) \phi_{s_{z}}\right]
\end{aligned}
$$

The final expression for the amplitude for the ${ }^{3} S_{1}$ state is given by

$$
\begin{aligned}
\mathcal{M}\left[{ }^{3} S_{1}^{(1)}\right]\left(P_{h}, k\right)= & \frac{1}{4 \sqrt{\pi M}} R_{0}(0) \frac{\delta_{a b}}{\sqrt{N_{c}}} \\
& \times \operatorname{Tr}\left[\sum_{m=1}^{3} O_{m}(0)\left(-\not P_{h}+M\right) \phi_{s_{z}}\right],
\end{aligned}
$$

where

$$
\begin{aligned}
& \sum_{m=1}^{3} O_{m}(0)=g_{s}^{2}\left(e e_{c}\right) \varepsilon_{\lambda_{g}}^{\rho *}\left(p_{g}\right)\left[\frac{\gamma_{\nu}\left(\not \not_{h}-2 \not 1+M\right) \gamma_{\mu}\left(-\not \not_{h}-2 \not p_{g}+M\right) \gamma_{\rho}}{\left(\hat{s}-M^{2}\right)\left(\hat{u}-M^{2}+q^{2}\right)}+\frac{\gamma_{\rho}\left(\not \not_{h}+2 \not p_{g}+M\right) \gamma_{\nu}\left(-\not p_{h}+2 \not k+M\right) \gamma_{\mu}}{\left(\hat{s}-M^{2}\right)\left(\hat{t}-M^{2}\right)}\right. \\
& \left.+\frac{\gamma_{\nu}\left(\not \not_{h}-2 \not 1+M\right) \gamma_{\rho}\left(-\not P_{h}+2 \not k+M\right) \gamma_{\mu}}{\left(\hat{t}-M^{2}\right)\left(\hat{u}-M^{2}+q^{2}\right)}\right] \text {. }
\end{aligned}
$$

\section{B. Calculation of the asymmetry}

We use a framework based on the generalized parton model, with the inclusion of intrinsic transverse momentum effects. We assume TMD factorization for the process considered. We consider a kinematical region in which the transverse momentum of $J / \psi$ is small compared to the mass of $J / \psi, M$, i.e. $P_{h \perp}<M$. The final gluon carries the momenta fraction $(1-z)$, as $z=\frac{P \cdot P_{h}}{P \cdot q}$ is the energy fraction transferred from the photon to $J / \psi$ in the proton rest frame. So, this means that when $z \rightarrow 1$, the outgoing gluon is soft. We consider $z<0.9$ to keep the final gluon hard. Moreover, gluon and heavy quark fragmentation can also contribute to quarkonium production significantly for $P_{h \perp}>4 \mathrm{GeV}$. We have imposed an upper limit on $P_{h \perp}$. In order to eliminate the fragmentation of the hard gluon into $J / \psi$ we also use a lower bound on $z$, namely $0.3<z$.

In the differential cross section given in Eq. (12), there is a contraction of four tensors which is written as

$$
L^{\mu \mu^{\prime}}(l, q) \Phi^{\nu \nu^{\prime}}\left(x, \boldsymbol{k}_{\perp}\right) \mathcal{M}_{\mu \nu}^{\gamma^{*}+g \rightarrow J / \psi+g} \mathcal{M}_{\mu^{\prime} \nu^{\prime}}^{* \gamma^{*}+g \rightarrow J / \psi+g},
$$

where the individual components are defined above. The summation over the transverse polarization of the final on-shell gluon is given by

$$
\begin{aligned}
& \sum_{\lambda_{a}=1}^{2} \varepsilon_{\mu}^{\lambda_{a}}\left(p_{g}\right) \varepsilon_{\mu^{\prime}}^{* \lambda_{a}}\left(p_{g}\right) \\
& \quad=-g_{\mu \mu^{\prime}}+\frac{p_{g \mu} n_{g \mu^{\prime}}+p_{g \mu^{\prime}} n_{g \mu}}{p_{g} \cdot n_{g}}-\frac{p_{g \mu} p_{g \mu^{\prime}}}{\left(p_{g} \cdot n_{g}\right)^{2}}
\end{aligned}
$$

with $n_{g}^{\mu}=\frac{P_{h}^{\mu}}{M}$. We have three amplitudes and their corresponding conjugates, given by Eq. (27), which will contribute. We use the notation

$$
\begin{aligned}
\mathcal{M}_{i}\left[{ }^{3} S_{1}^{(1)}\right]\left(P_{h}, k\right) \\
=\frac{1}{4 \sqrt{\pi M}} R_{0}(0) \frac{\delta_{a b}}{\sqrt{N_{c}}} \operatorname{Tr}\left[O_{i}(0)\left(-\not P_{h}+M\right) \phi_{s_{z}}\right]
\end{aligned}
$$

where $i=1,2,3$, corresponds to the contribution from each independent diagram.

So, the cross section will get a contribution from nine terms (of the form $M_{i} M_{j}$, where $i, j=1,2,3$ )

$$
M_{i} M_{j}=L^{\mu \mu^{\prime}}(l, q) \Phi^{\nu \nu^{\prime}}\left(x, \boldsymbol{k}_{\perp}\right) \mathcal{M}_{i \mu \nu}^{\gamma^{*}+g \rightarrow J / \psi+g} \mathcal{M}_{j \mu^{\prime} \nu^{\prime}}^{* \gamma^{*}+g \rightarrow J / \psi+g}
$$


and hence, the differential cross section can be written as

$$
\begin{aligned}
d \sigma= & \frac{1}{2 s} \frac{d^{3} l^{\prime}}{(2 \pi)^{3} 2 E_{l^{\prime}}} \frac{d^{3} P_{h}}{(2 \pi)^{3} 2 E_{P_{h}}} \int \frac{d^{3} p_{g}}{(2 \pi)^{3} 2 E_{g}} \\
& \times \int d x d^{2} \boldsymbol{k}_{\perp}(2 \pi)^{4} \delta\left(q+k-P_{h}-p_{g}\right) \frac{1}{Q^{4}}|M|^{2},
\end{aligned}
$$

where $M=\sum_{i} M_{i}$. Out of the nine terms in $|M|^{2}$, six are interference terms with a symmetry $M_{i} M_{j}=M_{j} M_{i}$ for $i \neq j$. So, effectively we need to compute six terms.

In a frame where the virtual photon and target proton move along the $z$-axis, and the lepton scattering plane defines the azimuthal angles $\phi_{l}=\phi_{l^{\prime}}=0$, then we have

$$
\begin{aligned}
& \frac{d^{3} l^{\prime}}{(2 \pi)^{3} 2 E_{l^{\prime}}}=\frac{1}{16 \pi^{2}} s y d x_{B} d y, \\
& \frac{d^{3} P_{h}}{(2 \pi)^{3} 2 E_{h}}=\frac{1}{(2 \pi)^{3}} \frac{1}{2 z} d z d^{2} \mathbf{P}_{h \perp} \\
& \frac{d^{3} p_{g}}{(2 \pi)^{3} 2 E_{g}}=\frac{1}{(2 \pi)^{3}} \frac{1}{2 z_{2}} d z_{2} d^{2} \mathbf{p}_{g \perp}
\end{aligned}
$$

and the delta function can be expressed as

$$
\begin{aligned}
\delta^{4}(q+ & \left.k-P_{h}-p_{g}\right) \\
= & \delta\left(x-\frac{1}{y s}\left(x_{B} y s+\frac{M^{2}+P_{h \perp}^{2}}{z}+\frac{\left(k_{\perp}-P_{h \perp}\right)^{2}}{(1-z)}\right)\right) \\
& \times \frac{2}{y s} \delta\left(1-z-z_{2}\right) \times \delta^{2}\left(\mathbf{k}_{\perp}-\mathbf{P}_{h \perp}-\mathbf{p}_{g \perp}\right),
\end{aligned}
$$

where the delta function sets $z_{2}=(1-z)$. Hence, after integrating with respect to $x, z_{2}$ and $p_{g \perp}$, the final form of the differential cross section can be given by

$\frac{d \sigma}{d y d x_{B} d z d^{2} \mathbf{P}_{h \perp}}=\frac{1}{256 \pi^{4}} \frac{1}{x_{B}^{2} s^{3} y^{2} z(1-z)} \int k_{\perp} d k_{\perp}\left|M^{\prime}\right|^{2}$,

where $\left|M^{\prime}\right|^{2}=\int d \phi|M|^{2}$, and $k_{\perp}$ is the magnitude of $\mathbf{k}_{\perp}$. As we are interested in the small $x$ region, we neglect terms containing higher powers of $x_{B}$; also as $z<1$, we neglect terms containing higher powers of $z$ and kept up to $z^{2}$. We also keep terms only up to $\left(\frac{k_{\perp}^{2}}{M_{p}^{2}}\right)$. The leading terms in the numerator of the $\cos \left(2 \phi_{h}\right)$ asymmetry come only from the first Feynman diagram. These terms are given in the Appendix. The denominator of the $\cos \left(2 \phi_{h}\right)$ asymmetry, which is defined below, is simply the cross section integrated over the azimuthal angle $\phi_{h}$. The leading terms in the cross section come from the $f_{1}^{g}$ term. All the terms corresponding to $h_{1}^{\perp g}$ are suppressed by $k_{\perp}^{2} / M_{p}^{2}$. Hence, from the approximations we mentioned above, the leading terms in the cross section in the denominator of $\cos \left(2 \phi_{h}\right)$ asymmetry are coming from $M_{1} M_{1}, M_{1} M_{2}, M_{1} M_{3}$. These contributions are given in the Appendix.

The differential cross section then can be given as

$$
\begin{aligned}
& \frac{d \sigma}{d y d x_{B} d z d^{2} \mathbf{P}_{h T}} \\
& =\frac{1}{256 \pi^{4}} \frac{1}{x_{B}^{2} s^{3} y^{2} z(1-z)} \\
& \quad \times \int k_{\perp} d k_{\perp}\left\{\left(A_{0}+A_{1} \cos \phi_{h}\right) f_{1}^{g}\left(x, \mathbf{k}_{\perp}^{2}\right)\right\} \\
& \quad+\frac{k_{\perp}^{2}}{M_{p}^{2}}\left\{\left(B_{0}+B_{1} \cos \phi_{h}+B_{2} \cos 2 \phi_{h}\right) h_{1}^{\perp g}\left(x, \mathbf{k}_{\perp}^{2}\right)\right\} .
\end{aligned}
$$

The coefficients $A_{0}, A_{1}, B_{0}, B_{1}$ and $B_{2}$ are given in the Appendix. The $\cos (2 \phi)$ asymmetry is defined as

$$
\left\langle\cos \left(2 \phi_{h}\right)\right\rangle=\frac{\int d \phi_{h} \cos \left(2 \phi_{h}\right) d \sigma}{\int d \phi_{h} d \sigma} .
$$

In order to estimate the $\cos \left(2 \phi_{h}\right)$ asymmetry, we need to parametrize the TMDs. We discuss two parametrization models, Gaussian parametrization of the TMDs and the McLerran-Venugopalan (MV) model. We also calculate the upper bound of the asymmetry.

\section{Gaussian parametrization of the TMDs}

Both for the linearly polarized gluon distribution and the unpolarized gluon TMD, a Gaussian parametrization is used widely in the literature. The linearly polarized gluon distribution satisfies the model independent positivity bound [1]:

$$
\frac{\mathbf{k}_{\perp}^{2}}{2 M_{p}^{2}}\left|h_{1}^{\perp g}\left(x, \mathbf{k}_{\perp}^{2}\right)\right| \leq f_{1}^{g}\left(x, \mathbf{k}_{\perp}^{2}\right) .
$$

The Gaussian parametrizations satisfy the positivity bound but do not saturate it. They are as follows [30-32]:

$$
\begin{gathered}
f_{1}^{g}\left(x, \mathbf{k}_{\perp}^{2}\right)=f_{1}^{g}(x, \mu) \frac{1}{\pi\left\langle k_{\perp}^{2}\right\rangle} e^{-k_{\perp}^{2} /\left\langle k_{\perp}^{2}\right\rangle} \\
h_{1}^{\perp g}\left(x, \mathbf{k}_{\perp}^{2}\right)=\frac{M_{p}^{2} f_{1}^{g}(x, \mu)}{\pi\left\langle k_{\perp}^{2}\right\rangle^{2}} \frac{2(1-r)}{r} e^{1-\frac{k_{\perp}^{2}}{r\left(k_{\perp}^{2}\right\rangle}},
\end{gathered}
$$

where $r(0<r<1)$ is a parameter, in our numerical estimates we took $r=1 / 3 . f_{1}^{g}(x, \mu)$ is the gluon collinear pdf, which is measured at the scale $\mu=\sqrt{M^{2}+P_{h \perp}^{2}}$ and it obeys the Dokshitzer-Gribov-Lipatov-Altarelli-Parisi 
(DGLAP) scale evolution. The width of the Gaussian, $\left\langle k_{\perp}^{2}\right\rangle$, depends on the energy scale of the process. Following [30], we took $\left\langle k_{\perp}^{2}\right\rangle=0.25 \mathrm{GeV}^{2}$. The asymmetry increases on increasing model parameter $r$, reaches a maximum at $r \approx 0.4$ and then decreases, but the variation of asymmetry is very small.

\section{Upper bound of the asymmetry}

The asymmetry reaches its maximum value when the positivity bound given by Eq. (38) is saturated. Using this, we calculate the upper bound of $\left|\left\langle\cos \left(2 \phi_{h}\right)\right\rangle\right|$ as below [19]:

$$
\begin{aligned}
\left|\left\langle\cos \left(2 \phi_{h}\right)\right\rangle\right| & =\left|\frac{\int d \phi_{h} \cos \left(2 \phi_{h}\right) d \sigma}{\int d \phi_{h} d \sigma}\right| \\
& =\frac{\int k_{\perp} d k_{\perp} \mathbf{k}_{\perp}^{2}\left|h_{1}^{\perp g}\left(x, \mathbf{k}_{\perp}^{2}\right)\right|}{\int k_{\perp} d k_{\perp} 2 M_{p} f_{1}^{g}\left(x, \mathbf{k}_{\perp}^{2}\right)} \frac{\left|B_{2}\right|}{A_{0}} \leq \frac{\left|B_{2}\right|}{A_{0}} \equiv R .
\end{aligned}
$$

\section{E. McLerran-Venugopalan (MV) model}

In the small $x$ region, the Weizsäcker-Williams (WW)type gluon distribution can be calculated in the MV model [44-46]: Within the nonperturbative McLerranVenugopalan model, we can define the gluon distribution function inside an unpolarized large nucleus or inside an energetic proton, in the small $x$ limit. In this model, the analytical expression of the WW-type unpolarized and linearly polarized gluon distributions are given by

$$
f_{1}^{g}\left(x, \mathbf{k}_{\perp}^{2}\right)=\frac{S_{\perp} C_{F}}{\alpha_{s} \pi^{3}} \int d r \frac{J_{0}\left(k_{\perp} r\right)}{r}\left(1-e^{-\frac{r^{2}}{4} Q_{s g}^{2}(r)}\right)
$$

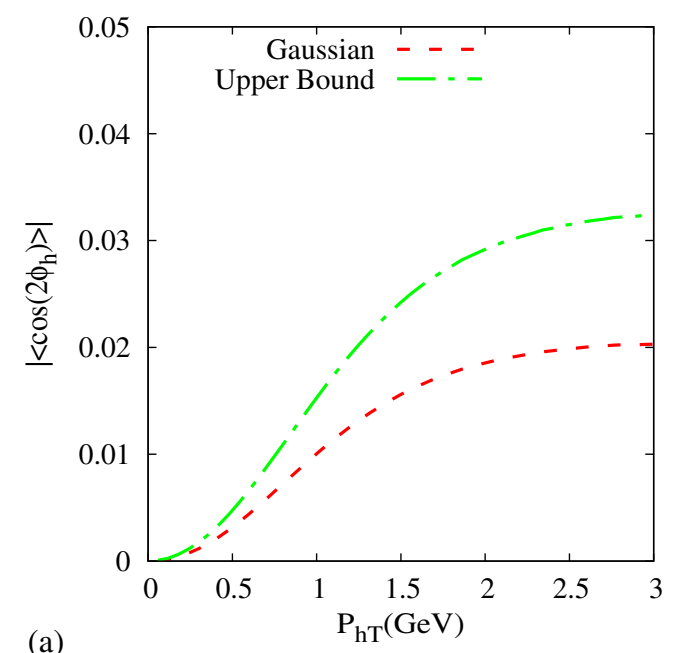

$h_{1}^{\perp g}\left(x, \mathbf{k}_{\perp}^{2}\right)=\frac{S_{\perp} C_{F}}{\alpha_{s} \pi^{3}} \frac{2 M_{p}^{2}}{k_{\perp}^{2}} \int d r \frac{J_{2}\left(k_{\perp} r\right)}{r \log \left(\frac{1}{r^{2} \Lambda_{\mathrm{QCD}}^{2}}\right)}\left(1-e^{-\frac{r^{2}}{4} Q_{s g}^{2}(r)}\right)$,

where $S_{\perp}$ is transverse size of the nucleus or nucleon. $Q_{s g}$ is the saturation scale, which in the MV model is defined as $Q_{s g}^{2}=\alpha_{s} N_{c} \mu_{A} \ln \frac{1}{r^{2} \Lambda_{\mathrm{QCD}}^{2}}$ and $\mu_{A} S_{\perp}=\alpha_{s} 2 \pi A$, where $A=1$ for the proton. Following the approach of [47], we have used a regularized version of the MV model in our calculation of the asymmetry. The ratio of linearly polarized and unpolarized distribution in the MV model can be given by

$\frac{\mathbf{k}_{\perp}^{2}}{2 M_{p}^{2}} \frac{h_{1}^{\perp g}\left(x, \mathbf{k}_{\perp}^{2}\right)}{f_{1}^{g}\left(x, \mathbf{k}_{\perp}^{2}\right)}=\frac{\int d r \frac{J_{2}\left(k_{\perp} r\right)}{r \log \left(\frac{1}{r^{2} \Lambda_{\mathrm{QCD}}^{2}}\right)}\left(1-e^{-\frac{r^{2}}{4} Q_{s g 0}^{2} \log \left(\frac{1}{r^{2} \Lambda_{\mathrm{QCD}}^{2}}\right)}\right)}{\int d r \frac{J_{0}\left(k_{\perp} r\right)}{r}\left(1-e^{-\frac{r^{2}}{4} Q_{s g 0}^{2} \log \left(\frac{1}{r^{2} \Lambda_{\mathrm{QCD}}^{2}}\right)}\right)}$.

For $Q_{s g 0}^{2}=\left(N_{c} / C_{F}\right) \times Q_{s 0}^{2}$, where $Q_{s 0}^{2}=0.35 \mathrm{GeV}^{2}$ at $x=0.01$ and $\Lambda_{\mathrm{QCD}}=0.2 \mathrm{GeV}$, the ratio is below 1 for all $k_{\perp}$. Below we give our numerical results.

\section{NUMERICAL RESULTS}

We have estimated the $\cos \left(2 \phi_{h}\right)$ asymmetry in $J / \psi$ production in the kinematics of EIC. MSTW2008 [48] is used for collinear pdfs. We have used the DGLAP evolution for the collinear pdfs. We have not included TMD evolution. As stated in the Introduction, we have used cuts on $z, 0.3<z<0.9$. As we know, gluon initiated processes are enhanced at small $x$. In fact, small $x$ values

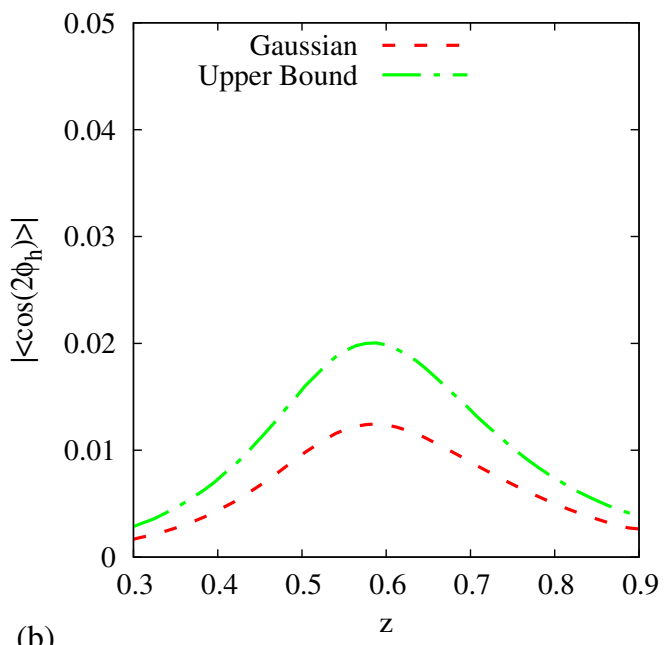

(b)

FIG. 2. $\cos \left(2 \phi_{h}\right)$ asymmetry in the $e+p \rightarrow e+J / \psi+X$ process as a function of (a) $P_{h T}$ (left panel) and (b) $z$ (right panel) at $\sqrt{s}=45 \mathrm{GeV}$ (EIC) and $x_{B}=0.01$. The integration ranges are $0<P_{h T} \leq 3 \mathrm{GeV}, 0.3<z<0.9$ and $0.05<y<0.4$. For convention of lines see the legend in the plots. 

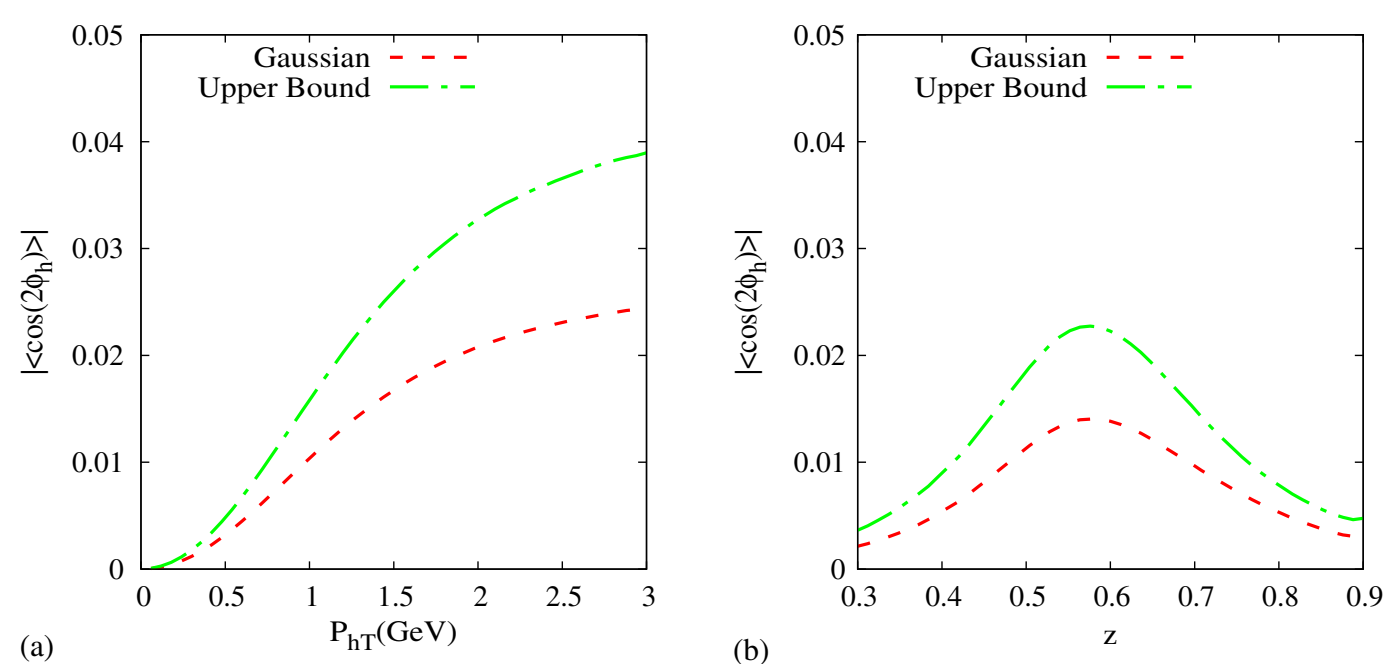

FIG. 3. $\cos \left(2 \phi_{h}\right)$ asymmetry in the $e+p \rightarrow e+J / \psi+X$ process as a function of (a) $P_{h T}$ (left panel) and (b) $z$ (right panel) at $\sqrt{s}=150 \mathrm{GeV}$ (EIC) and $x_{B}=0.01$. The integration ranges are $0<P_{h T} \leq 3 \mathrm{GeV}, 0.3<z<0.9$ and $0.005<y<0.04$. For convention of lines see the legend in the plots.

will be accessed at EIC, and this kinematical region will be very important in determining the gluon TMDs including the linearly polarized gluon TMD. In this work, we have studied the $\cos 2 \phi$ asymmetry for EIC in the small $x$ region. It is to be noted that $x$ is related to the Bjorken variable $x_{B}$ through Eq. (9). Smaller $x$ values also restrict $Q^{2}$ to be small; in this work we took $Q^{2}$ to be of the same order and bounded by $M^{2}\left(1<Q^{2}<9 \mathrm{GeV}^{2}\right)$, which is the mass of $J / \psi$. For both parametrizations used, the asymmetry is negative, which is consistent with the LO calculation [20]. In the plots, we show the magnitude of the asymmetry. CS LDMEs can be found for example in [49]. As only one state contributes in the CS model, namely ${ }^{3} S_{1}$, the asymmetry does not depend on the specific set of LDMEs. This is different from the $\mathrm{CO}$ model, where even at LO, contribution comes from several states [20], and the result depends on the choice of LDMEs. However, the unpolarized cross section will depend on the choice of LDMEs in both models. In our previous work [20], we compared three sets of LDMEs, where one set of LDMEs gives the unpolarized cross section that matches more with the experimental data than the other sets. Figures 2-4 show the upper bound of the asymmetry as well as an estimate using the Gaussian model, at $\sqrt{s}=45 \mathrm{GeV}, 150 \mathrm{GeV}$ and $190 \mathrm{GeV}$ respectively, as a function of $P_{h T}$ and $z$. Corresponding $x_{B}$ values are $x_{B}=0.01,0.01$ and 0.005 respectively; ranges of $y$ integration are $0.05<y<$ $0.4,0.005<y<0.04$ and $0.006<y<0.05$ respectively. $y$ is constrained by the choice of $Q^{2}$ and $x_{B}$. The transverse momentum $P_{h \perp}$ of $J / \psi$ is taken in the range

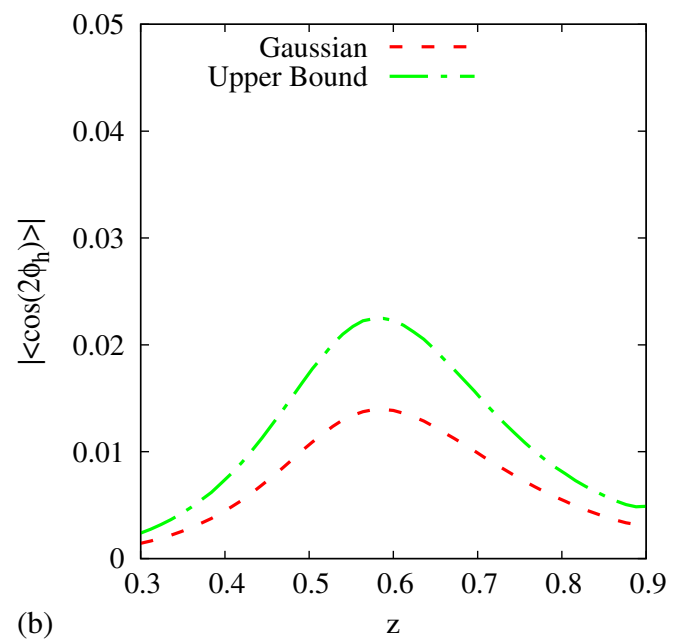

FIG. 4. $\cos \left(2 \phi_{h}\right)$ asymmetry in the $e+p \rightarrow e+J / \psi+X$ process as a function of (a) $P_{h T}$ (left panel) and (b) $z$ (right panel) at $\sqrt{s}=190 \mathrm{GeV}$ (EIC) and $x_{B}=0.005$. The integration ranges are $0<P_{h T} \leq 3 \mathrm{GeV}, 0.3<z<0.9$ and $0.006<y<0.05$. For convention of lines see the legend in the plots. 

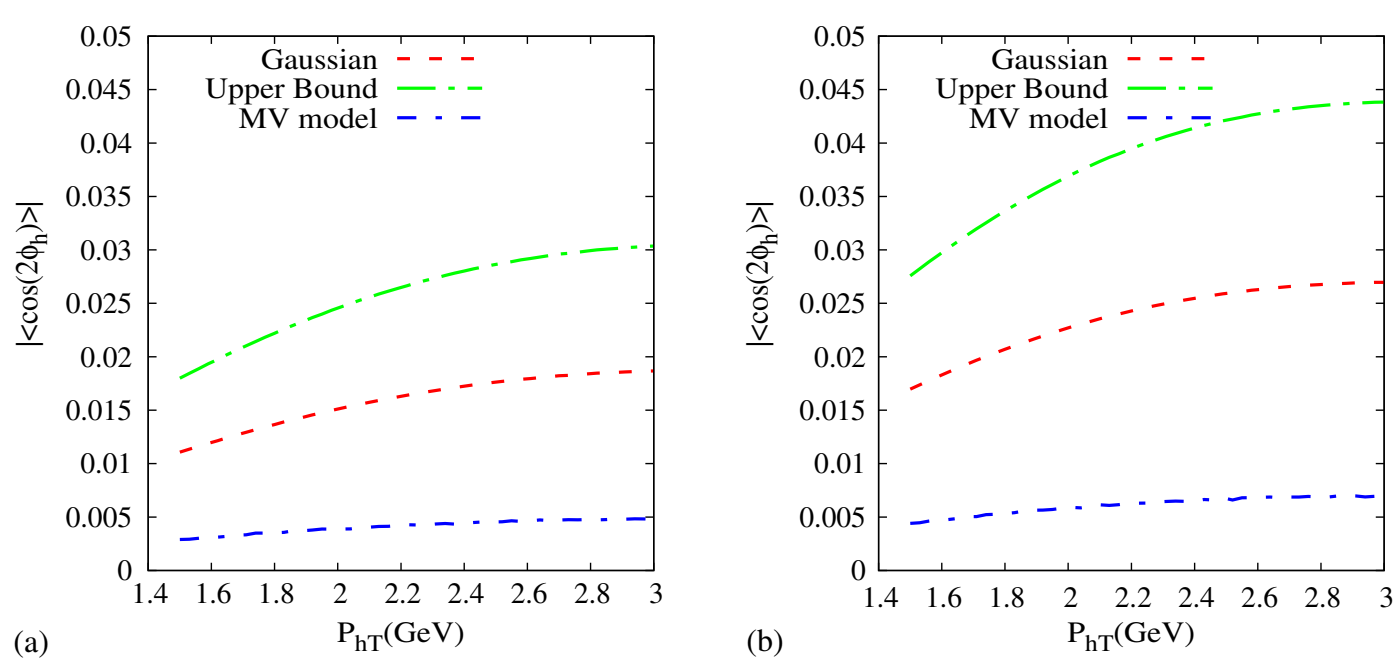

FIG. 5. $\cos \left(2 \phi_{h}\right)$ asymmetry in the $e+p \rightarrow e+J / \psi+X$ process as a function of $P_{h T}$ for (a) fixed $Q^{2}=9 \mathrm{GeV}^{2}, x=0.01$ and $z=0.5$ and (b) fixed $Q^{2}=9 \mathrm{GeV}^{2}, x=0.01$ and $z=0.7$. In both cases integration range on $y$ is $0.2<y<1$. For convention of lines see the legend in the plots.

$0<P_{h \perp}<3 \mathrm{GeV}$. The energy fraction $z$ is in the range $0.3<z<0.9$ for all of these plots. The upper bound of the asymmetry increases with an increase of $\sqrt{s}$ for the same $x_{B}$, it reaches maximum near $P_{h T} \approx 3 \mathrm{GeV}$, the maximum is about $4 \%$ for $\sqrt{s}=150 \mathrm{GeV}$. However, for smaller $x_{B}$, asymmetry decreases. The asymmetry reaches a peak near $z=0.6$ for the kinematical cuts chosen. The qualitative behavior of the asymmetry remains the same for all $\sqrt{s}$. The Gaussian model gives smaller asymmetry. Figure 5 shows a comparison of the upper bound of the asymmetry with that calculated in the Gaussian model as well as the MV model, as a function of $P_{h T}$, for two different values of $z$, (a) $z=0.5$ and (b) $z=0.7$. For both of these plots we have taken a fixed value of $x=0.01, Q^{2}=9 \mathrm{GeV}^{2}$ and $0.2<y<1$. For (a) $\sqrt{s}$ is in the range 61 to $181 \mathrm{GeV}$, and for (b) $\sqrt{s}$ is in the range 58 to $182 \mathrm{GeV}$. Asymmetry in the MV model is smaller compared to the Gaussian model, and both lie below the upper bound. The asymmetry is higher for higher values of $z$.

\section{CONCLUSION}

In this work, we have calculated the $\cos 2 \phi$ asymmetry in electroproduction of $J / \psi$ at EIC, which probes the linearly polarized gluon distribution in the unpolarized proton. We calculated the asymmetry in the kinematical region $z<1$, where the NLO subprocess $\gamma^{*}+g \rightarrow J / \psi+g$ gives the leading contribution. The gluon TMDs probed in this process are of Weizsäcker-Williams (WW) type. As gluon distributions play an important role in the small $x$ region, we investigate the asymmetry in the small $x$ kinematical region, using a Gaussian parametrization of the TMDs as well as in the McLerran-Venugopalan model. We also show the upper bound of the asymmetry saturating the inequality for the linearly polarized gluon distribution. At EIC, low values of $x$ also restrict the $Q^{2}$ (virtuality of the photon) values. We have calculated the $J / \psi$ production amplitude in the NRQCD based color singlet (CS) approach. The asymmetry in the kinematical region considered is small but sizable. The magnitude of the asymmetry may depend on the production mechanism of the quarkonium. As shown in [20], the CS mechanism underestimates the $J / \psi$ production at HERA, and both $\mathrm{CS}$ and $\mathrm{CO}$ contributions are needed to describe the data. In $\mathrm{CO}$ formalism contribution will come from several LDMEs in the final state, which may enhance the asymmetry. We plan to see the effect of the $\mathrm{CO}$ mechanism on the asymmetry in a future work. Another interesting study would be the effect of small- $x$ evolution on the asymmetry. In any case, the $\cos 2 \phi$ asymmetry in $J / \psi$ production at the EIC will be an important tool to gain information on the WW-type linearly polarized gluon distribution.

\section{ACKNOWLEDGMENTS}

We thank P. J. Mulders, E. Petreska and D. Boer for discussion. Part of the work of A. M. was done at NIKHEF, Amsterdam; the visit was supported by the European Research Council under the "Ideas" program QWORK (Contract No. 320389).

\section{APPENDIX: AMPLITUDES}

All the amplitude squares and the coefficients are integrated over $\phi$, where $\phi$ and $\phi_{h}$ are the azimuthal angle of initial gluon and $J / \psi$ respectively:

$$
M_{i}^{\prime} M_{j}^{\prime}=\int d \phi M_{i} M_{j}
$$




$$
\begin{aligned}
& M_{1}^{\prime} M_{1}^{\prime}=\left\{f_{1}^{g} \times 128 \pi M^{4}\left\{M^{4}(z-1)^{3}+M^{2}\left(P_{h \perp}^{2}(z-1)(8(z-1) z+3)+s y\left(x _ { B } \left(z \left((1-6 y(y+2)) z^{2}\right.\right.\right.\right.\right.\right. \\
& \left.\left.+2 y(y+4) z-2 y+z+1)-1)-x(z-1)^{3}\right)\right)+2 P_{h \perp} \sqrt{x_{B}} \sqrt{1-y} z \sqrt{s y} \cos \left(\phi_{h}\right)\left(M^{2}(z(4 z-5)+2)\right. \\
& \left.+P_{h \perp}^{2}(z(24 z-17)+4)-2 \operatorname{sxy}(z(4 z-3)+1)\right)+P_{h \perp}^{4}(z(z(11 z-23)+13)-3) \\
& +P_{h \perp}^{2} \operatorname{sy}\left(x(z(-2(z-5) z-9)+3)+x_{B}\left(z\left(-3(y(5 y+16)-6) z^{2}+2(y(y+14)-5) z-6 y+7\right)-3\right)\right) \\
& \left.+s^{2} x x_{B} y^{2} z(z((y-2) y(2 z-1)+2(8 z-7))+4)\right\}+\frac{k_{\perp}^{2} h_{1}^{\perp g}}{M_{p}^{2}} \times 64 \pi M^{4}\left\{M^{4}\left(-(z-1)^{3}\right)\right. \\
& +M^{2}\left(P_{h \perp}^{2}(z(-4(z-2) z-7)+3)+s y\left(x(z-1)^{3}+x_{B} z\left((y(7 y+18)-7) z^{2}-2 y(2 y+5) z\right.\right.\right.
\end{aligned}
$$

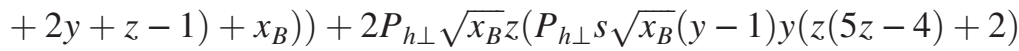

$$
\begin{aligned}
& \times \cos \left(2 \phi_{h}\right)-\sqrt{1-y} \sqrt{s y} \cos \left(\phi_{h}\right)\left(M^{2}(1-3 z)^{2}+P_{h \perp}^{2}(z(13 z-12)+5)\right. \\
& +\operatorname{sxy}(z(6 z-5)+2)))+P_{h \perp}{ }^{4}(z((7-3 z) z-6)+3)+P_{h \perp}^{2} s y\left(x _ { B } \left(z \left((3 y(3 y+8)-22) z^{2}-2(y(y+13)-8) z\right.\right.\right. \\
& +10 y-9)+3)-x(z(2 z(z+1)-5)+3))-s^{2} x y^{2} z\left(x\left(6 z^{2}-4 z+1\right)+x_{B} z((y-2) y(4 z-3)\right. \\
& \left.\left.\left.+26 z-20)-2 x_{B}(y-3)\right)\right\}\right\} /\left\{x y^{2}(z-1)^{2} z\left(M^{2}+s y\left(x_{B}-x\right)\right)^{2} \times\left(M^{2}+P_{h \perp}^{2}-s x_{B} y(z-2) z\right)^{2}\right\}
\end{aligned}
$$

$$
\begin{aligned}
& M_{1}^{\prime} M_{2}^{\prime}=\left\{f_{1}^{g} \times 64 \pi M^{6}\left\{M^{2}(z-1)(2 z-3)+P_{h \perp}^{2}(2 z(4 z-9)+9)-2 P_{h \perp} \sqrt{x_{B}} \sqrt{1-y}(z-1) z(3 z-8) \sqrt{s y} \cos \left(\phi_{h}\right)\right.\right. \\
& \left.-s y(z-1)\left(x(z+1)(2 z-3)+x_{B} z\left(3(y(y+6)-6) z^{2}-6(y(y+4)-3) z+4 y+3\right)+2 x_{B} y+x_{B}\right)\right\} \\
& -\frac{k_{\perp}^{2} h_{1}^{\perp g}}{M_{p}^{2}} \times 32 \pi M^{6}\left\{M^{2}(z-1)(2 z-3)+P_{h \perp}^{2}(2 z(3 z-7)+7)\right. \\
& -2 P_{h \perp} \sqrt{x_{B}} \sqrt{1-y}(z-1)(2 z-3)(3 z+1) \sqrt{s y} \cos \left(\phi_{h}\right)-s y(z-1)\left(x(3 z-5)+x_{B} z\left((y(5 y+6)-6) z^{2}\right.\right. \\
& \left.\left.\left.-2 y(5 y+7) z+2 y(y+2)+6 z+5)+x_{B}(4 y-1)\right)\right\}\right\} /\left\{s x^{2} y^{3}(z-1) z^{3}\right. \\
& \left.\times\left(M^{2}+s y\left(x_{B}-x\right)\right)^{2}\left(M^{2}+P_{h \perp}^{2}-s x_{B} y(z-2) z\right)\right\}
\end{aligned}
$$

$$
\begin{aligned}
& M_{1}^{\prime} M_{3}^{\prime}=\left\{-f_{1}^{g} \times 64 \pi M^{8}\left\{2 M^{2}(z-1)^{2}(3 z-5)+4 P_{h \perp}^{2}(z-1)(z(6 z-13)+8)\right.\right. \\
& -2 P_{h \perp} \sqrt{x_{B}} \sqrt{1-y}(z-1)^{2}(z(14 z-25)+2) \sqrt{s y} \cos \left(\phi_{h}\right)+s y\left(-x(3 z-10)(z-1)^{2}\right. \\
& +x_{B} z\left(y^{2}(z(z(31 z-57)+43)-9)+2 y(z(z(31 z-59)+53)-23)-2 z(5 z(5 z-8)+39)+56\right) \\
& \left.\left.-2 x_{B}((y-4) y+10)\right)\right\}+\frac{k_{\perp}^{2} h_{1}^{\perp g}}{M_{p}^{2}} \times 32 \pi M^{8}\left\{2 M^{2}(z-1)^{2}(7 z-9)+2 P_{h \perp}^{2}(z-1)(z(14 z-41)+29)\right. \\
& -4 P_{h \perp \sqrt{x_{B}}} \sqrt{1-y}(z-1)^{2}(7 z(2 z-3)+3) \sqrt{s y} \cos \left(\phi_{h}\right)+s y\left(x _ { B } \left(y^{2}(z(z(z(55 z-79)+45)-3)-4)\right.\right. \\
& +2 y(z(z(z(59 z-96)+76)-30)+5)+2 z(z((51-43 z) z-44)+37)-30) \\
& \left.\left.\left.-x(z-1)^{2}(3 z(4 z+1)-14)\right)\right\}\right\} /\left\{s x^{2} y^{3}(z-1)^{2} z^{2}\left(M^{2}+s y\left(x_{B}-x\right)\right)^{2}\right. \\
& \left.\times\left(M^{2}+P_{h \perp}^{2}-s x_{B} y(z-2) z\right)^{2}\right\}
\end{aligned}
$$

and the coefficients in final expression of cross section, Eq. (36), are as follows: 


$$
\begin{aligned}
& A_{0}=64 \pi M^{4}\left\{\left\{M ^ { 2 } ( z - 1 ) ( M ^ { 2 } + P _ { h \perp } ^ { 2 } - s x _ { B } y ( z - 2 ) z ) \left(M^{2}(z-1)(2 z-3)+P_{h \perp}^{2}(2 z(4 z-9)+9)\right.\right.\right. \\
& \left.-s y(z-1)\left(x(z+1)(2 z-3)+x_{B} z\left(3(y(y+6)-6) z^{2}-6(y(y+4)-3) z+4 y+3\right)+2 x_{B} y+x_{B}\right)\right) \\
& +M^{4} z\left(-2 M^{2}(z-1)^{2}(3 z-5)-4 P_{h \perp}^{2}(z-1)(z(6 z-13)+8)+\operatorname{sy}\left(x(3 z-10)(z-1)^{2}\right.\right. \\
& +x_{B} z\left((50-31 y(y+2)) z^{3}+(y(57 y+118)-80) z^{2}-y(43 y+106) z+y(9 y+46)+78 z-56\right) \\
& \left.\left.\left.+2 x_{B}((y-4) y+10)\right)\right)\right\} / s+2 x y z^{2}\left(M^{4}(z-1)^{3}+M^{2}\left(P_{h \perp}^{2}(z-1)(8(z-1) z+3)\right.\right. \\
& \left.+s y\left(x_{B}\left(z\left((1-6 y(y+2)) z^{2}+2 y(y+4) z-2 y+z+1\right)-1\right)-x(z-1)^{3}\right)\right)+P_{h \perp}^{4}(z(z(11 z-23)+13)-3) \\
& +P_{h \perp}^{2} s y\left(x(z(-2(z-5) z-9)+3)+x_{B}\left(z\left(-3(y(5 y+16)-6) z^{2}+2(y(y+14)-5) z-6 y+7\right)-3\right)\right) \\
& \left.\left.+s^{2} x x_{B} y^{2} z(z((y-2) y(2 z-1)+2(8 z-7))+4)\right)\right\} /\left\{x^{2} y^{3}(z-1)^{2} z^{3}\right. \\
& \left.\times\left(M^{2}+s y\left(x_{B}-x\right)\right)^{2}\left(M^{2}+P_{h \perp}^{2}-s x_{B} y(z-2) z\right)^{2}\right\} \\
& A_{1}=-128 \pi M^{4} P_{h \perp} \sqrt{x_{B}} \sqrt{1-y}\left\{-2 M^{4}(z-1)^{2}(7(z-2) z+5)+M^{2}\left(P_{h \perp}^{2}(z-1)^{2}(3 z-8)-s y z(2 x z(z(4 z-5)+2)\right.\right. \\
& \left.\left.+x_{B}(z-2)(3 z-8)(z-1)^{2}\right)\right)+2 \operatorname{sxy} z^{2}\left(P_{h \perp}^{2}((17-24 z) z-4)\right. \\
& +2 \operatorname{sxy}(z(4 z-3)+1))\} /\left\{x^{2} y^{2}(z-1)^{2} z^{2} \sqrt{s y}\left(M^{2}+s y\left(x_{B}-x\right)\right)^{2} \times\left(M^{2}+P_{h \perp}^{2}-s x_{B} y(z-2) z\right)^{2}\right\} \\
& B_{0}=32 \pi M^{4}\left\{\left\{-M^{2}(z-1)\left(M^{2}+P_{h \perp}^{2}-s x_{B} y(z-2) z\right)\left(M^{2}(z-1)(2 z-3)+P_{h \perp}^{2}(2 z(3 z-7)+7)\right.\right.\right. \\
& \left.-s y(z-1)\left(x(3 z-5)+x_{B} z\left((y(5 y+6)-6) z^{2}-2 y(5 y+7) z+2 y(y+2)+6 z+5\right)+x_{B}(4 y-1)\right)\right) \\
& +M^{4} z\left(2 M^{2}(z-1)^{2}(7 z-9)+2 P_{h \perp}^{2}(z-1)(z(14 z-41)+29)+s y\left(x _ { B } \left(y^{2}(z(z(z(55 z-79)+45)-3)-4)\right.\right.\right. \\
& \left.\left.\left.+2 y(z(z(z(59 z-96)+76)-30)+5)+2 z(z((51-43 z) z-44)+37)-30)-x(z-1)^{2}(3 z(4 z+1)-14)\right)\right)\right\} / s \\
& -2 x y z^{2}\left(M^{4}(z-1)^{3}+M^{2}\left(P_{h \perp}^{2}(z-1)(4(z-1) z+3)-s y\left(x(z-1)^{3}+x_{B} z\left((y(7 y+18)-7) z^{2}\right.\right.\right.\right. \\
& \left.\left.-2 y(2 y+5) z+2 y+z-1)+x_{B}\right)\right)+P_{h \perp}{ }^{4}(z(z(3 z-7)+6)-3)+P_{h \perp}{ }^{2} s y(x(z(2 z(z+1)-5)+3) \\
& \left.+x_{B}\left(z\left((22-3 y(3 y+8)) z^{2}+2(y(y+13)-8) z-10 y+9\right)-3\right)\right)+s^{2} x y^{2} z\left(x\left(6 z^{2}-4 z+1\right)\right. \\
& \left.\left.\left.+x_{B} z((y-2) y(4 z-3)+26 z-20)-2 x_{B}(y-3)\right)\right)\right\} /\left\{x^{2} y^{3}(z-1)^{2} z^{3}\right. \\
& \left.\times\left(M^{2}+s y\left(x_{B}-x\right)\right)^{2}\left(M^{2}+P_{h \perp}^{2}-s x_{B} y(z-2) z\right)^{2}\right\} \\
& B_{1}=-64 \pi M^{4} P_{h \perp} \sqrt{x_{B}} \sqrt{1-y}\left\{M^{4}(z-1)^{2}(z(4 z(7 z-12)+13)+3)\right. \\
& +M^{2}\left(\operatorname{syz}\left(2 x(1-3 z)^{2} z^{2}+x_{B}(z-2)(2 z-3)(3 z+1)(z-1)^{2}\right)-P_{h \perp}^{2}(z-1)^{2}(2 z-3)(3 z+1)\right) \\
& \left.+2 \operatorname{sxy} z^{3}\left(P_{h \perp}^{2}(z(13 z-12)+5)+\operatorname{sxy}(z(6 z-5)+2)\right)\right\} /\left\{x^{2} y^{2}(z-1)^{2} z^{3} \sqrt{s y}\left(M^{2}+s y\left(x_{B}-x\right)\right)^{2}\right. \\
& \left.\times\left(M^{2}+P_{h \perp}^{2}-s x_{B} y(z-2) z\right)^{2}\right\}
\end{aligned}
$$$$
B_{2}=\left\{128 \pi M^{4} P_{h \perp}{ }^{2} s x_{B}(y-1)(z(5 z-4)+2)\right\} /\left\{x y(z-1)^{2} \times\left(M^{2}+s y\left(x_{B}-x\right)\right)^{2}\left(M^{2}+P_{h \perp}^{2}-s x_{B} y(z-2) z\right)^{2}\right\} .
$$

[1] P. J. Mulders and J. Rodrigues, Phys. Rev. D 63, 094021 (2001).

[2] J.-P. Lansberg, C. Pisano, F. Scarpa, and M. Schlegel, Phys. Lett. B 784, 217 (2018).

[3] M. G. A. Buffing, A. Mukherjee, and P. J. Mulders, Phys. Rev. D 88, 054027 (2013).

[4] Y. V. Kovchegov and A. H. Mueller, Nucl. Phys. B529, 451 (1998).
[5] L. D. McLerran and R. Venugopalan, Phys. Rev. D 59, 094002 (1999).

[6] F. Dominguez, J.-W. Qiu, B.-W. Xiao, and F. Yuan, Phys. Rev. D 85, 045003 (2012).

[7] E. Iancu, A. Leonidov, and L. McLerran, arXiv:hep-ph/ 0202270.

[8] D. Kharzeev, Y. V. Kovchegov, and K. Tuchin, Phys. Rev. D 68, 094013 (2003). 
[9] D. Boer, S. Cotogno, T. van Daal, P. J. Mulders, A. Signori, and Y.-J. Zhou, J. High Energy Phys. 10 (2016) 013.

[10] F. Dominguez, B.-W. Xiao, and F. Yuan, Phys. Rev. Lett. 106, 022301 (2011).

[11] A. Metz and J. Zhou, Phys. Rev. D 84, 051503 (2011).

[12] A. Schafer and J. Zhou, Phys. Rev. D 88, 014008 (2013).

[13] E. Akcakaya, A. Schfer, and J. Zhou, Phys. Rev. D 87, 054010 (2013).

[14] A. Dumitru, T. Lappi, and V. Skokov, Phys. Rev. Lett. 115, 252301 (2015).

[15] C. Marquet, C. Roiesnel, and P. Taels, Phys. Rev. D 97, 014004 (2018).

[16] F. Dominguez, C. Marquet, B.-W. Xiao, and F. Yuan, Phys. Rev. D 83, 105005 (2011).

[17] D. Boer, P. J. Mulders, J. Zhou, and Y.-j. Zhou, J. High Energy Phys. 10 (2017) 196.

[18] S. Meissner, A. Metz, and K. Goeke, Phys. Rev. D 76, 034002 (2007).

[19] C. Pisano, D. Boer, S. J. Brodsky, M. G. A. Buffing, and P. J. Mulders, J. High Energy Phys. 10 (2013) 024.

[20] A. Mukherjee and S. Rajesh, Eur. Phys. J. C 77, 854 (2017).

[21] D. Boer, P. J. Mulders, and C. Pisano, Phys. Rev. D 80, 094017 (2009).

[22] A. V. Efremov, N. Ya. Ivanov, and O. V. Teryaev, Phys. Lett. B 777, 435 (2018).

[23] A. V. Efremov, N. Y. Ivanov, and O. V. Teryaev, Phys. Lett. B 780, 303 (2018).

[24] J.-P. Lansberg, C. Pisano, and M. Schlegel, Nucl. Phys. B920, 192 (2017).

[25] A. Dumitru, V. Skokov, and T. Ullrich, Phys. Rev. C 99, 015204 (2019).

[26] P. Sun, B.-W. Xiao, and F. Yuan, Phys. Rev. D 84, 094005 (2011).

[27] D. Boer, W. J. den Dunnen, C. Pisano, and M. Schlegel, Phys. Rev. Lett. 111, 032002 (2013).

[28] D. Boer, W. J. den Dunnen, C. Pisano, M. Schlegel, and W. Vogelsang, Phys. Rev. Lett. 108, 032002 (2012).
[29] M. G. Echevarria, T. Kasemets, P. J. Mulders, and C. Pisano, J. High Energy Phys. 07 (2015) 158; 05 (2017) 73.

[30] D. Boer and C. Pisano, Phys. Rev. D 86, 094007 (2012).

[31] A. Mukherjee and S. Rajesh, Phys. Rev. D 95, 034039 (2017).

[32] A. Mukherjee and S. Rajesh, Phys. Rev. D 93, 054018 (2016).

[33] G. T. Bodwin, E. Braaten, and G. P. Lepage, Phys. Rev. D 51, 1125 (1995); 55, 5853(E) (1997).

[34] G. P. Lepage, L. Magnea, C. Nakhleh, U. Magnea, and K. Hornbostel, Phys. Rev. D 46, 4052 (1992).

[35] C. E. Carlson and R. Suaya, Phys. Rev. D 14, 3115 (1976).

[36] E. L. Berger and D. L. Jones, Phys. Rev. D 23, 1521 (1981).

[37] R. Baier and R. Ruckl, Phys. Lett. 102B, 364 (1981).

[38] R. Baier and R. Ruckl, Nucl. Phys. B201, 1 (1982).

[39] E. Braaten and S. Fleming, Phys. Rev. Lett. 74, 3327 (1995).

[40] P. L. Cho and A. K. Leibovich, Phys. Rev. D 53, 150 (1996).

[41] P. L. Cho and A. K. Leibovich, Phys. Rev. D 53, 6203 (1996).

[42] U. D’Alesio, F. Murgia, C. Pisano, and P. Taels, Phys. Rev. D 96, 036011 (2017).

[43] S. Rajesh, R. Kishore, and A. Mukherjee, Phys. Rev. D 98, 014007 (2018).

[44] L. D. McLerran and R. Venugopalan, Phys. Rev. D 49, 2233 (1994).

[45] L. D. McLerran and R. Venugopalan, Phys. Rev. D 49, 3352 (1994).

[46] L. D. McLerran and R. Venugopalan, Phys. Rev. D 50, 2225 (1994).

[47] A. Bacchetta, D. Boer, C. Pisano, and P. Taels, arXiv: 1809.02056.

[48] A. D. Martin, W. J. Stirling, R. S. Thorne, and G. Watt, Eur. Phys. J. C 63, 189 (2009).

[49] K.-T. Chao, Y.-Q. Ma, H.-S. Shao, K. Wang, and Y.-J. Zhang, Phys. Rev. Lett. 108, 242004 (2012). 